TIFR-TH-95/61

hep-th/9511218

\title{
BLACK HOLE GEOMETRY AROUND AN ELEMENTARY BPS STRING STATE
}

\author{
Gautam Mandal and Spenta R. Wadia \\ Tata Institute of Fundamental Research \\ Homi Bhabha Road, Bombay 400 005, INDIA \\ e-mail: mandal, wadia@theory.tifr.res.in
}

\begin{abstract}
We probe the geometry around an elementary BPS (EBPS) state in heterotic string theory compactified on a six-torus by scattering a massless scalar off it and comparing with the corresponding experiment in which the EBPS state is replaced by a classical extremal black hole background satisfying the BPS condition. We find that the low energy limit of the scattering amplitudes precisely agree if one takes the limit $m_{\mathrm{bh}}>>m_{P}$. In the classical experiment, beyond a certain frequency of the incident wave, part of the wave is found to be absorbed by the black hole, whereas in case of the string scattering there is a critical frequency (inelastic threshold) of the probe beyond which the EBPS state gets excited to a higher mass non-BPS elementary state. The classical absorption threshold matches exactly with the inelastic threshold in the limit of maximum degeneracy of the EBPS state of a given mass. In that limit we can therefore identify absorption by the black hole as excitation of the elementary string state to the next vibrational state of the string and consequently also identify the non-BPS string state as a non-extremal black hole.
\end{abstract}




\section{Introduction}

It is well-known that elementary particles heavier than the Planck mass have Compton wavelength smaller than their classical 'Schwarzschild radius'. The suggestion that such particles may be identified with black holes and conversely black holes may be viewed as elementary particles has a long history [1]. Stable excitations of such masses abound in $N=2$ string compactifications, and if the above identification is true, it might have profound effect on our understanding of both black hole physics and string theory. Recently there have been a number of papers [2, 3, 4, 5, 6, 7] investigating from various viewpoints the correspondence between classical extremal (BPS) black hole solutions in toroidally compactified heterotic string and elementary BPS states in the same theory carrying the same mass and charge as the black hole.

In this paper we probe the geometry around an elementray BPS (EBPS) state by the following 'experiments'. We choose as our probe a massless scalar excitation of the string theory (we work with heterotic string compactified on a six-torus throughout). We scatter this probe off (a) the extremal black hole solution and (b) the EBPS state in flat space carrying the same quantum numbers (mass and charge). The idea is that if the background created by the EBPS state is the same as that of the classical solution, the results should agree. Here are the main results:

(i) The classical scattering calculation involves solving the wave equation of the massless scalar (of frequency $w$ ) in the black hole background and calculating the phase shifts $\delta_{l}$ of the partial waves (Sec 1). The string scattering (Sec 2) involves calculating the tree-level four-point amplitude< EBPS-probe-probe-EBPS $\rangle$. The low energy limit of this amplitude agrees (Sec 3) precisely with the leading classical result if one further takes the limit $m>>m_{P}$ lin the string calculation, where $m$ is the mass of the EBPS state (equal to the ADM mass of the black hole).

(ii) The string scattering calculation explicitly involves internal polarization tensors which appear in the vertex operator for the EBPS state, whereas these do not appear in the specification of the classical background. In the limit mentioned above any nontrivial dependence of the string amplitude on

\footnotetext{
${ }^{1}$ We are working in Einstein units rather than string units, so the natural scale in our problem is $m_{P}=1 / \sqrt{\alpha^{\prime}}$ rather than $m_{s}=g_{s} m_{P}$.
} 
the 'hair' (internal polarization tensors of the EBPS state) disappears (Sec 2).

(iii) In the classical calculation there exists a critical frequency $w_{c r}$ such that when the frequency $w$ of the massless scalar wave exceeds $w_{c r}$ the phase shifts $\delta_{l}$ start becoming complex and the incoming wave gets partly absorbed by the black hole. On the string side there exists a critical frequency $w_{c r}^{\prime}$ such that for $w \geq w_{c r}^{\prime}$ the EBPS state can get excited to a non-BPS elementary state. These two critical frequencies exactly coincide when the ratio $\left|Q_{L}\right| /\left|Q_{R}\right| \rightarrow 0\left(Q_{L}, Q_{R}\right.$ are the left- and right-moving charges) which is also the limit of maximum allowed degeneracy of the EBPS state of a given mass. In that limit we are therefore led to a rather simple interpretation of absorption of matter by the black hole as simply excitation of the string state to a higher vibrational mode. It also implies an identification of these non-BPS states with non-extremal black hole. We remark on the issue of the de-excitation process and Hawking radiation in the concluding section (Sec. 4).

\section{Classical Scattering}

We consider the extremal black hole solution in heterotic string theory compactified on a six-torus [3]. We reproduce some of the formulae we will need here. The low energy 4D lagrangian describing the dynamics of the massless fields is

$S=\frac{1}{16 \pi G_{N}} \int d^{4} x \sqrt{-g}\left[R_{g}-e^{-\Phi} F_{\mu \nu}^{(a)}(L M L)_{a b} F^{(b) \mu \nu}+\frac{1}{8} \operatorname{Tr}\left(\partial_{\mu} M L \partial^{\mu} M L\right)+\ldots\right]$

where $G_{N}$ is Newton's constant and equals $\alpha^{\prime} / 8 . g_{\mu \nu}$ is the Einstein metric and indices are contracted above with respect to it. $M$ is a $28 \times 28$ matrixvalued field representing the massless scalars. $L$ is a $28 \times 28$ diagonal matrix with the first 22 entries equal to -1 and the remaining six equal to 1 . The terms denoted by ... do not involve $M$. The above lagrangian possesses a black hole solution [3] given by

$$
d s^{2} \equiv g_{\mu \nu} d x^{\mu} d x^{\nu}=-\rho / \sqrt{K} d t^{2}+\sqrt{K} / \rho d \rho^{2}+\rho \sqrt{K} d \Omega^{2}
$$


where

$$
\begin{aligned}
& K=\rho^{2}+2 m_{0} \cosh \alpha \rho+m_{0}^{2} \\
& \tanh \alpha=\left|Q_{L}\right| /\left|Q_{R}\right| \\
& m_{0}=\alpha^{\prime} m /(4 \cosh \alpha)
\end{aligned}
$$

We denote by $Q_{R}, Q_{L}$ the charge vectors in the right-moving and left-moving sectors (and by $\left|Q_{L, R}\right|$ their magnitudes) respectively. $m$ is the ADM mass of the black hole.

We consider the propagation of fluctuations of the massless scalar $\left(\delta M_{a b}\right)$ in such a background. The equation of motion is given by

$$
D^{\mu} \partial_{\mu} \delta M_{a b}=0
$$

This can be derived by putting $M_{a b}=M_{a b}^{\mathrm{cl}}+\delta M_{a b}$ in the above lagrangian. The terms linear in $\delta M$ vanish by classical equation of motion for the background $M^{\mathrm{cl}}$. The remaining terms involving $\delta M$ are quadratic and lead to (1.3). The metric appearing in the above equation is the 'Einstein' metric. We choose the scalars as probes because their propagation equation is particularly simple, involving only the 'Einstein' metric and no other background.

The classical scattering of $\delta M_{a b}$ off the black hole (1.1) is computed by doing the partial wave analysis of (1.3) and finding out the phase shifts as detailed below. We should remark that (1.3) is the same as the equation $L_{0}=\bar{L}_{0}=1$ for the vertex operator for $\delta M$ in the curved background. Consequently a scattering calculation using curved-space sigma-model worldsheet action and vertex operators (1.3) for the massless scalar string state is in principle equivalent to the following analysis.

Step 1. Partial wave analysis:

We look for solutions of the form ( $c f$. 8] )

$$
\begin{aligned}
& \delta M_{a b}(\rho, t, \theta, \phi)=M_{w}(\rho, \theta, \phi) \exp (-i w t) \\
& M_{w}(\rho, \theta, \phi)=\sum_{l} Y_{l 0}(\theta, \phi) \psi_{w l}(\rho) / \rho
\end{aligned}
$$

Substituting the above in (1.3) we find that the partial waves $\psi_{w l}$ satisfy the following equation:

$$
-\psi^{\prime \prime}+V(\rho) \psi=0, \quad V(\rho)=\frac{l(l+1)}{\rho^{2}}-w^{2} \frac{K}{\rho^{2}}
$$


This equation is exactly solvable in terms of confluent hypergemotric functions. The result is 12

$$
\psi(\rho)=\rho^{l^{\prime}+1} \exp [i w \rho]\left(A M\left(a, 2\left(l^{\prime}+1\right),-2 i w \rho\right)+B U\left(a, 2\left(l^{\prime}+1\right),-2 i w \rho\right)\right)
$$

Here

$$
\begin{aligned}
& l^{\prime}=-1 / 2+\sqrt{(l+1 / 2)^{2}-m_{0}^{2} w^{2}} \\
& a=l^{\prime}+1-i a^{\prime}, \quad a^{\prime} \equiv m_{0} \cosh \alpha w
\end{aligned}
$$

In the above $A$ and $B$ are integration constants. The requirement of finiteness of the solution at $\rho=0$ gives us the boundary condition (for $m_{0} w<1 / 2$ )

$$
B=0
$$

since $U \propto \rho^{-2 l^{\prime}-1}$ as $\rho \rightarrow 0$. Using the $\rho \rightarrow \infty$ asymptotics of the confluent hypergeometric functions we now get the phase shifts $\delta_{l}$ of the partial waves $\psi_{w l}$ :

$$
\begin{aligned}
& \psi_{w l}(\rho) \sim C_{w l} \sin \left(w \tilde{\rho}+\delta_{l}-\pi l / 2\right) \\
& \tilde{\rho} \equiv \rho+m_{0} \cosh \alpha \ln (2 w \rho) \\
& \exp \left(2 i \delta_{l}\right)=\Gamma\left(l^{\prime}+1-i a^{\prime}\right) / \Gamma\left(l^{\prime}+1+i a^{\prime}\right)
\end{aligned}
$$

The symbol $\sim$ throughout this paper will imply 'asymptotically true for large $\rho$.

Step 2. Summing up the partial waves:

In order to obtain the scattering amplitude we need to choose $C_{w l}$ in (1.9) such that the sum over partial waves in (1.4) is of the form $($ as $\rho \rightarrow \infty)$

$$
M_{w}(\rho, \theta, \phi) \sim \exp \left[i w\left(z-m_{0} \cosh \alpha \ln w(\rho-z)\right)\right]+\frac{f(\theta)}{\rho} \exp [i w \tilde{\rho}]
$$

This is done in Appendix A. The result for $f(\theta)$ is the following:

$$
f(\theta)=\frac{\alpha^{\prime} m}{8} \operatorname{cosec}^{2} \theta / 2\left[1+o\left(m_{0} w, w / m_{P}\right)\right]
$$

Note that the above scattering amplitude is independent of the 'polarization' $(a b)$ of $\delta M_{a b}$. We shall see that in the string calculation also the scattering 
amplitude becomes independent of the polarization of the massless scalar in a suitable limit.

Absorption Threshold: Note that the phase shifts $\delta_{l}$, given by (1.9), remain real as long as $m_{0} w<1 / 2$, that is, as long as

$$
w \leq w_{c r}, \quad w_{c r}=1 /\left(2 m_{0}\right)
$$

For $w>w_{c r}$, the phase shifts become complex, signalling absorption. One can explicitly calculate the flux through a small 2-sphere around $\rho=0$ and show that at $w>w_{c r}$ this becomes non-zero.

\section{$2 \quad$ String-string scattering}

We work with the heterotic string theory compactified on a six-dimensional torus. We will use the notation $x^{\mu}(z, \bar{z}), \mu=1, \ldots, 4$ for the 4 non-compact coordinates, $x_{R}^{i}(z), i=1, \ldots, 6$ for the 6 compact right-moving coordinates and $x_{L}^{j}(\bar{z}), j=1, \ldots, 22$ for the 22 compact left-moving coordinates. The right-moving fermions will be denoted by $\psi^{\mu}(z)$ and $\psi_{R}^{i}(z)$. To calculate the scattering of a massless scalar off an elementary BPS state we need the following ingredients:

(a) Vertex operator for the massless scalar $\left(M_{a b}\right)$ :

$$
\begin{aligned}
& \mathcal{V}_{M}\left(\eta_{R} ; \eta_{L} ; k ; z, \bar{z}\right)=V_{M}\left(\eta_{R}, k, z\right) \bar{V}_{M}\left(\eta_{L}, \bar{z}\right) \exp [i k . x(z, \bar{z})] \\
& V_{M}\left(\eta_{R}, k, z\right)=\eta_{R} \cdot\left(\partial_{z} x_{R}+i k_{\mu} \psi^{\mu} \psi_{R}\right) \\
& \bar{V}_{M}\left(\eta_{L}, \bar{z}\right)=\eta_{L} \cdot \partial_{\bar{z}} x_{L}
\end{aligned}
$$

(b) Vertex operator for the elementary BPS state:

The elementary BPS states satisfy a mass formula?

$$
m^{2}=\left|Q_{R}\right|^{2}=\left|Q_{L}\right|^{2}+2\left(N_{L}-1\right)
$$

where $Q_{R}, Q_{L}$ are the left-moving and right-moving charges. Given some mass $m, N_{L}$ is not fixed, so one has to consider various cases.

\footnotetext{
${ }^{2}$ In the rest of this section we will work with the convention $\alpha^{\prime}=2$.
} 
$\underline{\text { Case } N_{L}=1}$ : The vertex operator in this case is given by

$$
\begin{aligned}
& \mathcal{V}_{B}\left(\zeta_{R} ; \zeta_{L} ; k ; z, \bar{z}\right)=V_{B}\left(\zeta_{R}, k, z\right) \bar{V}_{B}\left(\zeta_{L}, \bar{z}\right) \exp \left[i Q_{R} \cdot x_{R}+i Q_{L} \cdot x_{L}+i k \cdot x(z, \bar{z})\right] \\
& V_{B}(\zeta, k, z)=\zeta_{R} \cdot \psi_{R}(z) e^{-\phi(z)} \\
& \bar{V}_{B}\left(\zeta_{L}, \bar{z}\right)=\zeta_{L} \cdot \partial_{\bar{z}} x_{L}
\end{aligned}
$$

We have chosen $V_{B}$ in the '-1' picture in order to provide $\phi$-ghost charge -2 in the following four-point function. We have also chosen polarization vectors of the EBPS state to lie only in the internal compact directions since we want to make correspondence with a spin-zero black hole.

We now consider scattering a probe (particle '2') off an EBPS state (particle ' 1 '). The final states will be denoted '4' and ' 3 ' respectively. We will call the initial and final polarizations of the probe $\eta_{L, R}, \eta_{L, R}^{\prime}$ and those of the EBPS state $\zeta_{L, R}, \zeta_{L, R}^{\prime}$. The four-point scattering amplitude is given by (computed in Appendix B)

$$
\begin{aligned}
& \mathcal{M}(1,2,3,4) \equiv \int \prod d^{2} z_{3}\left\langle c \bar{c}\left(z_{1}\right) c \bar{c}\left(z_{2}\right) c \bar{c}\left(z_{4}\right)\right. \\
& \left.\mathcal{V}_{B}\left(\zeta_{R} ; \zeta_{L} ; k_{1} ; z_{1}\right) \mathcal{V}_{M}\left(\eta_{R} ; \eta_{L} ; k_{2} ; z_{2}\right) \mathcal{V}_{B}^{(-)}\left(\zeta_{R}^{\prime} ; \zeta_{L}^{\prime} ; k_{3} ; z_{3}\right) \mathcal{V}_{M}\left(\eta_{R}^{\prime} ; \eta_{L}^{\prime} ; k_{4} ; z_{4}\right)\right\rangle \\
& =\left[K_{R}^{1}-\frac{t(t+2)}{\left(s-m^{2}\right)\left(u-m^{2}\right)} K_{R}^{2}-\frac{t(t+2)}{2\left(s-m^{2}\right)} K_{R}^{3}-\frac{t(t+2)}{2\left(u-m^{2}\right)} K_{R}^{4}\right] \times \\
& {\left[K_{L}^{1}-\frac{t(t+2)}{\left(s-m^{2}\right)\left(u-m^{2}\right)} K_{L}^{2}+\frac{t(t+2)}{\left(s-m^{2}\right)\left(s-m^{2}+2\right)} K_{L}^{3}+\frac{t(t+2)}{\left(u-m^{2}\right)\left(u-m^{2}+2\right)} K_{L}^{4}\right] A_{1}(s, t, u)}
\end{aligned}
$$

where

$$
A_{1}(s, t, u)=-\pi \Gamma\left(-\frac{t}{2}\right) \Gamma\left(\frac{m^{2}-u}{2}+1\right) \Gamma\left(\frac{m^{2}-s}{2}+1\right) /\left[\Gamma\left(\frac{t}{2}+2\right) \Gamma\left(\frac{u-m^{2}}{2}\right) \Gamma\left(\frac{s-m^{2}}{2}\right)\right]
$$

and

$$
\begin{array}{ll}
K_{R}^{1}=\eta_{R} \cdot \eta_{R}^{\prime} \zeta_{R} \cdot \zeta_{R}^{\prime}, & K_{R}^{2}=\zeta_{R} \cdot \zeta_{R}^{\prime} \eta_{R} \cdot Q_{R} \eta_{R}^{\prime} \cdot Q_{R} \\
K_{R}^{3}=\zeta_{R} \cdot \eta_{R} \zeta_{R}^{\prime} \cdot \eta_{R}^{\prime}, & K_{R}^{4}=\zeta_{R} \cdot \eta_{R}^{\prime} \zeta_{R}^{\prime} \cdot \eta_{R} \\
K_{L}^{1}=\eta_{L} \cdot \eta_{L}^{\prime} \zeta_{L} \cdot \zeta_{L}^{\prime}, & K_{L}^{2}=\zeta_{L} \cdot \zeta_{L}^{\prime} \eta_{L} \cdot Q_{L} \eta_{L}^{\prime} \cdot Q_{L} \\
K_{L}^{3}=\zeta_{L} \cdot \eta_{L} \zeta_{L}^{\prime} \cdot \eta_{L}^{\prime}, & K_{L}^{4}=\zeta_{L} \cdot \eta_{L}^{\prime} \zeta_{L}^{\prime} \cdot \eta_{L}
\end{array}
$$

In the above $\mathcal{V}_{B}^{(-)}$indicates that the corresponding vertex operator carries charge $-Q_{R},-Q_{L}$, ensuring charge conservation. Since our probe here is 
neutral, the left and right moving charges of the EBPS state cannot change in the scattering process.

In order to start comparing with the classical scattering, let us work in the rest frame of the initial EBPS state (particle 1). We use the following notation:

$$
k_{1}=(m, \overrightarrow{0}), k_{2}=(w, \vec{k}), k_{3}=\left(E^{\prime}, \vec{k}-\vec{k}^{\prime}\right), k_{4}=\left(w^{\prime}, \vec{k}^{\prime}\right) ; \vec{k} \cdot \vec{k}^{\prime} \equiv w w^{\prime} \cos \theta
$$

With this, $t=-4 w w^{\prime} \sin ^{2} \frac{\theta}{2}, s=m^{2}+2 w m$ and $u=m^{2}-2 m w+4 w w^{\prime} \sin ^{2} \frac{\theta}{2}$, where by momentum conservation, $w^{\prime}=w\left(1+2(w / m) \sin ^{2}(\theta / 2)\right)^{-1}$. It follows that for $w<<m_{P}$ (which implies $w<<m$ )

$$
A_{1}(s, t, u)=\pi \alpha^{\prime} \frac{m^{2}}{4} \operatorname{cosec}^{2} \frac{\theta}{2}[1+o(w / m)]
$$

and that the all terms in (2.4) other than the one containing the product $K_{R}^{1} K_{L}^{1}$ are either down by $w / m_{P}$ or they are down by $\left(m_{P} / m\right)^{2}$. Thus we get

$$
\mathcal{M}(1,2,3,4)=\pi \alpha^{\prime} \frac{m^{2}}{4} \operatorname{cosec}^{2} \frac{\theta}{2}\left[K_{1}+o(w / m)+o\left(m / m_{P}\right)^{2}\right]
$$

with

$$
K_{1}=K_{R}^{1} K_{L}^{1}
$$

In equations (2.8) and (2.9) we have reinstated $\alpha^{\prime}\left(=1 / m_{P}^{2}\right)$.

Case $N_{I}>1$ : The holomorphic part of the vertex operator for the EBPS state, $V_{B}(z)$, remains as above, while there are now many choices of the antiholomorphic part, corresponding to the various ways a state with a given $N_{L}>1$ can be constructed. Let us choose a basis $\bar{V}_{B}^{(r)}(\bar{z})$ for these vertex operators. In general these will satisfy an OPE:

$$
\bar{V}_{B}^{(r)}(\bar{z}) \bar{V}_{B}^{(s)}(\bar{w})=\frac{Z^{r s}}{(\bar{z}-\bar{w})^{2 N_{L}}}
$$

In the following (see the normalization convention in Appendix $\mathrm{C}$ ) we will use an orthonormal basis of vertex operators so as to make $Z^{r s}=\delta_{r s}$. We will denote the full vertex for the EBPS state as

$$
\mathcal{V}_{B}\left(\zeta_{R} ; r ; k ; z, \bar{z}\right)=V_{B}(\zeta, k, z) \bar{V}_{B}^{(r)}(\bar{z}) \exp \left[i Q_{R} . x_{R}+i Q_{L} \cdot x_{L}+i k . x(z, \bar{z})\right]
$$


Examples are: $\bar{V}_{B}^{(r)}(\bar{z})=\zeta_{L, i} \partial_{\bar{z}}^{2} x^{i}$ or $=\zeta_{L, i j} \partial_{\bar{z}} x^{i} \partial_{\bar{z}} x^{j}$ for $N_{L}=2$.

Let us denote the initial and final polarizations of the EBPS state as $\left(\zeta_{R}, r\right)$ and $\left(\zeta_{R}^{\prime}, s\right)$. The four-point amplitude is now given by (Appendix B)

$$
\begin{aligned}
& \mathcal{M}(1,2,3,4) \equiv \int d^{2} z_{3}\left\langle c \bar{c}\left(z_{1}\right) c \bar{c}\left(z_{2}\right) c \bar{c}\left(z_{4}\right)\right. \\
& \left.\mathcal{V}_{B}\left(\zeta_{R} ; r ; k_{1} ; z_{1},\right) \mathcal{V}_{M}\left(\eta_{R} ; \eta_{L} ; k_{2} ; z_{2}, \bar{z}_{2}\right) \mathcal{V}_{B}^{(-)}\left(\zeta_{R}^{\prime} ; s ; k_{3} ; z_{3}, \bar{z}_{3}\right) \mathcal{V}_{M}\left(\eta_{R}^{\prime} ; \eta_{L}^{\prime} ; k_{4} ; z_{4}, \bar{z}_{4}\right)\right\rangle \\
& =A_{1}(s, t, u)\left[K_{1}+o\left(w / m_{P}\right)+o\left(m / m_{P}\right)^{2}\right]
\end{aligned}
$$

$K_{1}$ is once again defined by (2.10) with $K_{L}^{1}$ this time given by

$$
K_{L}^{1}=\eta_{L} \cdot \eta_{L}^{\prime} Z^{r s}
$$

The low energy (and $m>>m_{P}$ ) limit of the amplitude $\mathcal{M}(1,2,3,4)$ is given once again by the equation (2.9).

No hair: Note that the internal polarization of the probe and the EBPS state are decoupled in the factor $K_{1}$ for both $N_{L}=1$ and $N_{L}>1$. By way of contrast, terms like $K_{R}^{3}$ (Eqn. (2.5)) have inner products between the internal polarizations of the probe and those of the EBPS state. Amplitudes involving the term $K_{R}^{3}$ can be used, by sending in probes in suitable internal state of polarization, to measure the state of internal polarization of the EBPS state, in contradiction to the no-hair theorem. However, as we have seen above, such terms disappear in the low energy limit.

Inelastic threshold: It is easy to deduce the inelastic threshold, namely $w_{c r}^{\prime}$, such that for $w>w_{c r}^{\prime}$ the probe can excite the EBPS state to the next higher mass state with the same charges $Q_{R}, Q_{L}$ (remember that the probe, being neutral, cannot change these charges). The general mass formula

$$
m^{2}=\left|Q_{R}\right|^{2}+2 N_{R}-1=\left|Q_{L}\right|^{2}+2 N_{L}-2
$$

(BPS condition is $N_{R}=1 / 2$ ) says that the next higher mass state (mass $m^{\prime}$ ) above the EBPS state is the one obtained by letting $N_{R} \rightarrow N_{R}+1, N_{L} \rightarrow$ $N_{L}+1$, so that

$$
m^{\prime 2}=m^{2}+2
$$

The state obtained by $N_{R} \rightarrow N_{R}+n, N_{L} \rightarrow N_{L}+n$ has a mass $m^{(n)}$ where

$$
\left[m^{(n)}\right]^{2}=m^{2}+2 n
$$


It is a simple exercise in relativistic kinematics to show that the state $m^{\prime}$ cannot be excited unless the probe has a frequency $w \geq w_{c r}^{\prime}$ " (in the rest frame of the original EBPS state) where

$$
w_{c r}^{\prime}=2 /\left(m \alpha^{\prime}\right)
$$

where we have reinstated $\alpha^{\prime}$.

\section{Comparison between String Scattering and Classical Scattering}

Scattering amplitude: The string amplitude $\mathcal{M}(1,2,3,4)$ leads to the following scattering cross-section (Appendix C) in the rest frame of ' 1 ':

$$
d \sigma / d \Omega=\frac{E_{4}^{2}}{E_{2}^{2} E_{1} E_{3}}|\mathcal{M}(1,2,3,4)|^{2}(2 \pi)^{-2}
$$

and $\sigma \equiv \int \sin \theta d \theta d \phi d \sigma / d \Omega$, where $\theta, \phi$ are the relative angles between the vectors $\vec{k}_{4}$ and $\vec{k}_{2}$. On the other hand, the cross-section in the classical scattering is given by

$$
d \sigma / d \Omega=|f(\theta)|^{2}
$$

If the geometry around the EBPS state is indeed correctly reproduced by the classical black hole solution, then the two scattering cross-sections should agree. Therefore we must have

$$
f(\theta)=\frac{E_{4}}{2 \pi E_{2} \sqrt{E_{1} E_{3}}} \mathcal{M}(1,2,3,4)
$$

upto a constant phase factor.

Now the low energy $w / m_{P} \rightarrow 0$ limit of (1.11) and (2.9) are, respectively,

$$
f(\theta)=\alpha^{\prime} \frac{m}{8} \operatorname{cosec}^{2} \frac{\theta}{2}
$$

and

$$
\mathcal{M}(1,2,3,4)=\alpha^{\prime} \frac{\pi m^{2}}{4} \operatorname{cosec}^{2} \frac{\theta}{2}\left[1+o\left(m / m_{P}\right)^{2}\right]
$$

\footnotetext{
${ }^{3}$ We have explicitly checked, for $w \geq w_{c r}^{\prime}$, that the tree level amplitude for the process $\mathrm{EBPS}+$ probe $\rightarrow$ non-BPS (mass $m^{\prime}$ ) + probe is non-zero.
} 
In the last equation we have used the fact that $K_{1}$ gives rise to 1 when we sum and average $\left|K_{1}\right|^{2}$ in (3.1) over final and initial polarization states respectively. Noting that to leading order in $w / m_{P}, E_{1}=E_{3}=m, \quad E_{2}=$ $E_{4}=w$, we find that the right hand side of (3.3) is

$$
\alpha^{\prime} \frac{m}{8} \operatorname{cosec}^{2} \frac{\theta}{2}\left[1+o\left(m / m_{P}\right)^{2}\right]
$$

which precisely agrees with (3.4) in the limit $m_{P} / m \rightarrow 0$.

We should note that in (1.11) there are correction terms of the form $w m_{0}=4\left(w / m_{P}\right)\left(m / m_{P}\right)$ which implies that we must first expand to leading order in $w / m_{P}$ before taking the large $m / m_{P}$ limit; alternatively we should define $w<<m_{P}\left(m_{P} / m\right)$ as the appropriate low-energy limit.

Disappearance of hair: As we remarked after (2.14), terms in the scattering amplitude which potentially constitute a measurement of the internal polarizations ('hair') of the EBPS state disappear in the same limit as discussed above.

Thresholds: It is trivial to see that the inelastic thrsehold is given by

$$
w_{c r}^{\prime}=2 / \alpha^{\prime} m=\cosh \alpha /\left(2 m_{0}\right)
$$

which agrees with the absorption threshold (1.12) in the limit $\cosh \alpha \rightarrow 1$, or equivalently $N_{L} \rightarrow N_{\max } \equiv 1+\alpha^{\prime} m^{2} / 4$ (note that by (2.2) or (2.15) this is the maximum possible value of $N_{L}$ for a given mass). Since the degeneracy of the BPS state essentially comes from the number of left-moving oscillators (in the right sector the BPS condition forces $N_{R}=1 / 2$ ), therefore $N_{L}=N_{\max }$ is also the limit when the degeneracy of the EBPS state, for a given mass, is maximized.

\section{Concluding Remarks}

We have seen that the geometry around the large mass EBPS state is the same as that of the classical extremal black hole solution as seen by a masslass scalar probe. The agreement has been verified to the leading order in the low energy expansion. It is interesting to note that a curved space sigma model description of string theory which is normally supposed to describe a 'string 
condensate' of massless modes represents a very high mass elementary string state in our scattering experiment.

It is also interesting to observe that the low energy limit $\left(w<<m_{P}\right)$ restores the no-hair theorem, since the terms capable of measuring the internal polarization state of the EBPS state (which is responsible for its degeneracy, given the mass and the charges) drop out in this limit. The interpretation of such terms in the case $w \approx m_{P}$ or in the case where the leading term in (2.9) is made to disappear by choosing the initial and final polarization of the probe to be orthogonal remains an interesting open problem.

Another intriguing result that we found is the emergence of the $N_{L} \rightarrow$ $N_{\text {max }}$ limit where the classical absorption threshold and the inelastic threshold of the string scattering agree. In a sense the test provided by this agreement is more stringent than the agreement of the scattering amplitude, since absorption is a rather essential feature of black hole geometry as against scattering in conventional central fields of force. Note that we need $N_{L}$ to be large also for the agreement [3] between the degeneracy of the EBPS state and the Beckenstein-Hawking entropy of the classical black hole (the degeneracy formulae do not hold for $N_{L}$ of order 1 ). We have already remarked that $N_{L}=N_{\max }$ corresponds to the maximum degeneracy of the EBPS state for a given mass. The existence of a limit where the two thresholds agree gives a rather simple picture in that limit of what happens when matter falls into the black hole. The black hole absorbs the energy and gets excited to a higher vibrational state of the string. This higher mass state is a nonBPS state and hence it decays according to standard string theory back to the BPS state. It would be extremely interesting to see under what circumstances such a decay might possibly correspond to Hawking radiation. Work in this direction is in progress.

Acknowledgement: We would like to thank A. Dhar and A. Sen for many useful discussions. G.M. would like to thank R.S. Bhalerao, S.S. Jha, S.M. Roy, K.V.L. Sarma and N. Ullah for discussions regarding scattering theory for the modified Coulomb problem.

Note added: After this paper was completed, the paper [13] was pointed out to us by A. Sen which has some overlap with this work. 


\section{A Derivation of the scattering amplitude $f(\theta)$}

The following sum over partial waves is well-known from the theory of scattering in a Coulomb potential [8]:

$$
\begin{aligned}
& \sum_{l=0}^{\infty}(2 l+1) i^{l} \exp \left[i \eta_{l}\right] P_{l}(\cos \theta) \sin \left[w \tilde{\rho}+\eta_{l}-l \pi / 2\right] /(w \rho) \\
& \sim \exp \left[i w\left(z-m_{0} \cosh \alpha \ln w(\rho-z)\right)\right]+\rho^{-1} f_{c}(\theta) \exp [i w \tilde{\rho}]
\end{aligned}
$$

where

$$
\begin{gathered}
\eta_{l}=\arg \Gamma\left(l+1-i a^{\prime}\right), l=0,1, \ldots, \infty \\
f_{c}(\theta)=(1 / 2) m_{0} \cosh \alpha \operatorname{cosec}^{2} \frac{\theta}{2} \exp i \varphi \\
\varphi=m_{0} w \cosh \alpha \ln \sin ^{2}(\theta / 2)+2 \eta_{0}
\end{gathered}
$$

and $a^{\prime}$ and $\tilde{\rho}$ are as defined in (1.7) and in (1.9). The logarithmic corrections to the phases of the incident and the outgoing waves are characteristic of any potential with a $1 / \rho$ fall-off at infinity. The above equation is meant to be true at asymptotically large distances $\rho$.

Since we have a modified Coulomb potential (1.5), our phase shifts $\delta_{l}$, determined in (1.9), differ from $\eta_{l}$. However, for small $m_{0} w$, it is easy to see that

$$
l^{\prime}=l-\left(m_{0} w\right)^{2} /(2 l+1)+o\left(m_{0} w\right)^{4}
$$

so that

$$
\delta_{l}=\eta_{l}+\Delta_{l}, \Delta_{l}=\frac{1}{2 l+1}\left[o\left(m_{0} w\right)^{2}\right]
$$

In order to arrive at a sum over partial waves analogous to (A.1) for our present problem we do the following manipulations [8]:

$$
\begin{aligned}
& (w \rho)^{-1} \sum_{l=0}^{\infty}(2 l+1) i^{l} \exp \left[i \delta_{l}\right] P_{l}(\cos \theta) \sin \left[w \tilde{\rho}+\delta_{l}-l \pi / 2\right] \\
& =(2 i w \rho)^{-1} \sum_{l}(2 l+1) i^{l} \exp \left[i \eta_{l}\right]\left(\left[\exp \left(2 i \Delta_{l}\right)-1\right] \exp [i w \tilde{\rho}] \exp i\left[\eta_{l}-l \pi / 2\right]+\right. \\
& \left.\quad \sin \left[w \tilde{\rho}+\eta_{l}-l \pi / 2\right]\right) P_{l}(\cos \theta) \\
& \sim \exp \left[i w\left(z-m_{0} \cosh \alpha \ln w(\rho-z)\right)\right]+\rho^{-1}\left[f_{c}(\theta)+f_{m}(\theta)\right] \exp [i w \tilde{\rho}],
\end{aligned}
$$




$$
f_{m}(\theta)=\frac{1}{w} \sum_{l}(2 l+1) \exp \left[2 i \eta_{l}\right] \frac{\exp \left[2 i \Delta_{l}\right]-1}{2 i} P_{l}(\cos \theta)
$$

Using (A.4) it is easy to see that the sum in (A.6) is convergent and $f_{m}(\theta)=$ $m_{0} \times\left[o\left(m_{0} w\right)\right]$. Noting further that $\varphi=o\left(m_{0} w\right)$ in $(\mathrm{A} .2)$, we find that (A.5) reduces to the right hand side of (1.10) with $f(\theta)$ given in (1.11). In order that $M_{w}$, given by (1.4) and (1.9), reduces to the left hand side of (A.5) we need to choose

$$
C_{w l}=\sqrt{4 \pi(2 l+1)} i^{l} \exp \left[2 i \delta_{l}\right] / w
$$

This concludes the derivation of (1.11). The additional $o\left(w / m_{P}\right)$ correction has been added in (1.11) because the metric itself can get corrected to the next order in $\alpha^{\prime}$, leading to such corrections.

\section{B Details of String Scattering}

We reproduce only the essential steps here. The basic method is the same as in [10].

\section{Case $N_{I}=1$}

The integrand in (2.4) reduces to a product $R L K$ where

$$
\begin{gathered}
R=\left\langle c V_{B}\left(z_{1}\right) \exp \left[i Q_{R} \cdot x_{R}\left(z_{1}\right)\right] c V_{M}\left(z_{2}\right) V_{B}\left(z_{3}\right) \exp \left[-i Q_{R} \cdot x_{R}\left(z_{3}\right)\right] c V_{M}\left(z_{4}\right)\right\rangle \\
L=\left\langle\bar{c} \bar{V}_{B}\left(\bar{z}_{1}\right) \exp \left[i Q_{L} \cdot x_{L}\left(\bar{z}_{1}\right)\right] \bar{c} \bar{V}_{M}\left(\bar{z}_{2}\right) \bar{V}_{B}\left(\bar{z}_{3}\right) \exp \left[-i Q_{L} \cdot x_{L}\left(\bar{z}_{3}\right)\right] \bar{c} \bar{V}_{M}\left(\bar{z}_{4}\right)\right\rangle \\
K=\left\langle\prod_{i} \exp \left[i k_{i} \cdot x\left(z_{i}, \bar{z}_{i}\right)\right]\right\rangle
\end{gathered}
$$

Before writing down the expressions for $R$ and $L$ let us, as in [10], use the $S L_{2}(C)$ invariance of the four-point amplitude to choose $z_{1}=0, z_{2}=1$ and $z_{4} \rightarrow \infty$. We will denote $z_{3}$ by $z$. The correlation functions in this notation evaluate to

$$
\begin{aligned}
R & =(-z)^{-m^{2}-2}\left[\zeta_{R} \cdot \zeta_{R}^{\prime} \eta_{R} \cdot \eta_{R}^{\prime}\left(1-k_{2} \cdot k_{4}\right)+z k_{2} \cdot k_{4} \zeta_{R} \cdot \eta_{R} \zeta_{R}^{\prime} \cdot \eta_{R}^{\prime}\right. \\
& \left.-\frac{z}{1-z} k_{2} \cdot k_{4} \zeta_{R} \cdot \eta_{R}^{\prime} \zeta_{R}^{\prime} \cdot \eta_{R}+\frac{z^{2}}{1-z} \zeta_{R} \cdot \zeta_{R}^{\prime} \eta_{R} \cdot Q_{R} \eta_{R}^{\prime} \cdot Q_{R}\right]
\end{aligned}
$$




$$
\begin{gathered}
L=(-\bar{z})^{-m^{2}-2}\left[\zeta_{L} \cdot \zeta_{L}^{\prime} \eta_{L} \cdot \eta_{L}^{\prime}+\bar{z}^{2} \zeta_{L} \cdot \eta_{L} \zeta_{L}^{\prime} \cdot \eta_{L}^{\prime}+\left(\frac{\bar{z}}{1-\bar{z}}\right)^{2} \zeta_{L} \cdot \eta_{L}^{\prime} \zeta_{L}^{\prime} \cdot \eta_{L}\right. \\
\left.-\frac{\bar{z}^{2}}{1-\bar{z}} \zeta_{L} \cdot \zeta_{L}^{\prime} \eta_{L} \cdot Q_{L} \eta_{L}^{\prime} \cdot Q_{L}\right] \\
K=|z|^{2 k_{1} \cdot k_{3}}|1-z|^{2 k_{2} \cdot k_{3}}
\end{gathered}
$$

We now integrate $R L K$ using the formula [11],

$$
\begin{aligned}
\int d^{2} z z^{a+n_{1}}(1-z)^{b+n_{2}} \bar{z}^{a+n_{3}}(1-\bar{z})^{b+n_{4}}=-\sin \pi b \times \\
B\left(a+n_{1}+1, b+n_{2}+1\right) B\left(-\left(a+b+n_{3}+n_{4}+1\right), b+n_{4}+1\right)
\end{aligned}
$$

We put $a=k_{1} \cdot k_{3}-m^{2}-2=-t / 2-2$ and $b=k_{2} \cdot k_{3}=\left(m^{2}-u\right) / 2$. By using $\Gamma(z+1)=z \Gamma(z)$ repeatedly we get (2.4).

Case $N_{L}>1$

The factors $R$ and $K$ remain the same. Our strategy for $L$ is as follows. It is not difficult to see that the $n_{3}=n_{4}=0$ term always gives rise to the same kinetic factor $\left(A_{1}(s, t, u)\right)$ as in the case $N_{L}=1$. Does the pattern that other values of $n_{3}, n_{4}$ give rise to integrals which are down either by $w / m_{P}$ or by $\left(m_{P} / m\right)^{2}$ persist? Indeed it does. Proof: the entire set of values of $n_{3}, n_{4}\left(n_{3} \geq 0, n_{4} \leq 0\right)$ can be classified in terms of the ratio

$$
f\left(n_{3}, n_{4}\right)=\frac{B\left(-\left(1+a+b+n_{3}+n_{3}\right), b+n_{4}+1\right)}{B(-(1+a+b), b+1)}
$$

as follows:

$$
\begin{array}{rll}
\left(n_{3}, n_{4}\right)=(0,0): & f=1 \\
n_{3} \in\{0,1\}, n_{4} \leq-1: & f=o(1 / m w) \\
\left(n_{3}, n_{4}\right)=(1,0): & f=o(1 / m w) \\
n_{3} \geq 2, n_{4}=0: & f=o(w / m) \\
n_{3}+n_{4} \geq 1, n_{4} \leq-1: & f=o\left(1 / m^{2}\right) \\
n_{3}+n_{4} \leq 0, n_{3} \geq 2: & f=o(w / m)
\end{array}
$$

It is not too difficult to see that contractions corresponding to the second and third lines do not appear in $L$ at all. The crucial thing to note is that there is no other term than the top line which survives the $w / m_{P} \rightarrow 0, m_{P} / m \rightarrow 0$ limit. This proves (2.16). 


\section{Normalization Convention for S-matrix}

We discuss here the relative coefficient between $S$-matrix elements and string scattering amplitudes. Suppose

$$
\begin{gathered}
\langle 3,4|-i S| 1,2\rangle=C_{0} \mathcal{M}(1,2,3,4)\left[\prod_{i} 2 w_{i} \Omega\right]^{-1 / 2}(2 \pi)^{4} \delta^{(4)}\left(\sum_{i} k_{i}\right) \\
\langle 3|-i S| 1,2\rangle=C_{0} \mathcal{M}(1,2,3)\left[\prod_{i} 2 w_{i} \Omega\right]^{-1 / 2}(2 \pi)^{4} \delta^{4}\left(\sum_{i} k_{i}\right)
\end{gathered}
$$

where $\mathcal{M}(1,2,3,4)$ is as defined in (2.4) and $\mathcal{M}(1,2,3)=\left\langle c \bar{c} V_{1} c \bar{c} V_{2} c \bar{c} V_{3}\right\rangle$. The vertex operators are normalized to satisfy an OPE: $V(z) V(w)=\mid z-$ $\left.w\right|^{-4}$. In the above equations we follow the convention and notation of [9], where $\Omega$ is the volume of space. The $(2 \pi)^{4} \delta^{(4)}\left(\sum_{i} k_{i}\right)$ stands for the integral $\int d^{4} x \exp [i k . x]$ over a space box of volume $\Omega$ and a time interval $T$. The constant $C_{0}$ can be fixed by demanding tree-level unitarity in some simple example. In these conventions $C_{0}$ turns out to be 4 .

With the above convention, the relation between the $S$-matrix element and the scattering cross-section (in the rest frame of particle ' 1 ') is [9]

$$
d \sigma=\sum_{3,4}|<3,4| S|1,2>|^{2} \times \Omega /\left(v_{2} T\right)
$$

where $v_{2}$ is the velocity of particle 2 in the rest frame of 1 . Using the standard manipulations of squaring a delta-function etc., it is straightforward to arrive at (3.1).

\section{References}

[1] S. Hawking, Monthly Notices Roy. Astron. Soc. 152 (1971) 75; A. Salam, in Quantum Gravity: an Oxford Symposium (eds. C. Isham, R. Penrose and D. Sciama, O.U.P. 1975); G. 'tHooft, Nucl. Phys. B335 (1990) 138; J. Preskill, Physica Scripta T36 (1991) 258; C. Holzhey and F. Wilczek, Nucl. Phys. B380 (1992) 447 (hep-th/9202014); L. Susskind, preprint RU-93-44 (hep-th/9309145); J. Russo and L. Susskind, Nucl. Phys. B437 (1995) 611 (hep-th/9405117); M. Duff, R. Khuri, R. Minasian and J. Rhamfeld, Nucl. Phys. B418 (1994) 195 (hep-th/9311120); see also references in [3]. 
[2] M. Duff and J. Rahmfeld, Phys. Lett. B $345 \quad$ (1995) 441 (hep-th/9406105).

[3] A. Sen, Mod. Phys. Lett. A 10 (1995) 2081 (hep-th/9504147).

[4] R. Khuri and R. Myers, preprint MCGILL-95-38, (hep-th/9508045).

[5] A. Dabholkar, J. Gauntlett, J. Harvey and D. Waldram, preprint CALT68-2028 (hep-th/9511053).

[6] F. Larsen and F. Wilczek, PUPT-1576 (hep-th/9511064).

[7] L. Susskind, preprint SU-ITP-95-23 (hep-th/9511116).

[8] L.I. Schiff, Quantum Mechanics (McGraw-Hill 1968); N.F. Mott and H.S.W. Massey, The Theory of Atomic Collisions (Oxford 1965).

[9] T.D. Lee, Particle Physics and Introduction to Field Theory (Harwood 1981).

[10] D. Friedan, E. Martinec and S. Shenker, Nucl. Phys. B 271 (1986) 93.

[11] M. Green, J. Schwarz and E. Witten, Superstring Theory: Volume 1 (Cambridge 1987); H. Kawai, D.C. Lewellen and S.-H.H. Tye, Nucl. Phys. B269 (1986) 1.

[12] M. Abramowicz and I. Stegun, Handbook of Mathematical Functions (Dover 1972).

[13] C.G. Callan, J.M. Maldacena and A.W. Peet, PUPT-1565 (hep-th/9510134). 